\title{
VoxSanguinis
}

$\mathrm{IF}=$ International Forum

$\mathrm{ABO}$ incompatibility

- pretransfusion bedside compatibility test 37

AIHA

- alloantibodies against red cells

- - detection (IF) 200

Alloantibodies against red cells

- AIHA

- - detection (IF) 200

Anti-HEV in blood donors (letter) 198

Antibody engineering (review) 72

Apheresis

- platelet-leukocyte interaction 101

Arterial blood gases and $\mathrm{pH}$

- rapid blood transfusion 158

Autologous blood donation, adolescents

- aggressive phlebotomy schedule 231

Bacteria and HIV in platelet concentrates

- photochemical indication 209

Bacterial contamination

- blood components (IF) 59

Blood donors, female

- haemoglobin levels 225

CD34+ and mononuclear cells in leukapheresis

- collection efficiencies 242

Coagulation factors, retention

- plasma frozen after $24 \mathrm{~h} 28$

$\mathrm{D}^{\mathrm{Va}}$ category pheno- and genotype

- Japan 194

Eicosanoids, plasma levels

- intraoperatively 'salvaged' blood 31

Evans antigen heterogeneity 44

FVIII or FIX antibodies in haemophiliacs

- optimal treatment (IF) 256

Fc function, IVIG

- flow cytometry 185

Flow cytometry

- IVIG, Fc function 185

\section{GBV C/HGV}

- liver disease 143
$\mathrm{HCV}$

- blood donors (letter) 254

- - TT virus infection (letter) 254

- in haemophiliacs and blood donors

- $\quad$ genotype analysis (letter) 255

- screening (PCR) (letter) 57

- Rh immunoglobulin 1

- RNA reference reagents

- - calibration 217

- RNA stability 137

Hepatitis $\mathrm{C}$ core antigen

- detection in 'window' phase 80

HIV and bacteria in platelet concentrates

- photochemical inactivation 209

HLA, soluble

- immunoadsorption 119

HTLV indeterminate donors

- prospective study (letter) 130

International Forum:

AIHA, detection of alloantibodies against RC 20

Bacterial contamination of blood components 59

FVIII or FIX antibodies in haemophiliacs

- optimal treatment 256

IVIG, Fc function

- flow cytometry 185

Letters to the Editor:

Anti-HEV in blood donors 198

$\mathrm{HCV}$

- blood donors

- _ TT virus infection 254

haemophiliacs and blood donors

- $\quad$ genotype analysis 255

PCR screening 57

HTLV indeterminate donors

- prospective study 130

Plasma for practical classes

- viral status 130

Platelet concentrations

- monocyte activation 133

Transfusion safety (multiple units) 132 Leukapheresis

- CD34+ and mononuclear cells

- - collection efficiencies 242 
Leukocyte-poor platelet concentration

- preparation 164

Leukodepleted platelets

- comparison 171

Liver disease

- GBV C/HGV 143

Lymphocytotoxic antibodies

- test 250

Monocyte activation

- platelet concentration (letter) 130

MNS blood group antigens

- Vr and $\mathrm{Mt}^{\mathrm{a}} 52$

Neutrophil elastase

- leukocyte depletion of blood components 19

Obituary:

Hässig, Alfred 69

Parvovirus B19, human

- PCR fractionation plasma 7

Peripheral blood stem cell transplantation

- thrombopoietin 106

$\mathrm{pH}$ and arterial blood gases

- rapid blood transfusion 158

Phage display (review) 72

Phosphate

- platelet additive solution 176

Photochemical inactivation

- bacteria and HIV in platelet concentrates 209

Plasma fractionation

- prion protein 86

Plasma for practical classes

- viral status (letter) 130

Platelet additive solution

- phosphate 176
Platelet concentration

- in-line filtration 235

- monocyte activation (letter) 133

Platelet leukocyte interaction

- apheresis 101

Prion protein

- plasma fractionation 86

$\mathrm{RBC}$ transfusion, allogeneic

- postoperative bacterial infection 13

Red cell aplasia, pure

- AIHA-associated 126

- - splenic lymphoma 126

$\mathrm{Rh}$ immunoglobulin

- safety 1

$\mathrm{RhD}^{\mathrm{Va}}$

- polymorphism (Japan) 122

Serological cross-match

- elimination 113

Splenic lymphoma

- AIHA-associated red cell aplasia 126

Surgery

- transfusion 96

Thrombopoietin

- peripheral blood stem cell transplantation 101

Transfusion in surgery 96

Transfusion safety, multiple units (letter) 132

TT virus

- HCV blood donors (letter) 254

Viral marker screening (1997)

- unpaid donors 149

Viral status

- plasma for practical classes (letter) 130 